\title{
On nabla conformable fractional Hardy-type inequalities on arbitrary time scales
}

\author{
Ahmed A. El-Deeb ${ }^{1 *}$ (D), Samer D. Makharesh', Eze R. Nwaeze², Olaniyi S. Iyiola ${ }^{3}$ and Dumitru Baleanu 4,5,6
}

\author{
"Correspondence: \\ ahmedeldeeb@azhar.edu.eg \\ 'Department of Mathematics, \\ Faculty of Science, Al-Azhar \\ University, Nasr City (11884), Cairo, \\ Egypt \\ Full list of author information is \\ available at the end of the article
}

\begin{abstract}
The main aim of the present article is to introduce some new $\nabla$-conformable dynamic inequalities of Hardy type on time scales. We present and prove several results using chain rule and Fubini's theorem on time scales. Our results generalize, complement, and extend existing results in the literature. Many special cases of the proposed results, such as new conformable fractional $h$-sum inequalities, new conformable fractional $q$-sum inequalities, and new classical conformable fractional integral inequalities, are obtained and analyzed.
\end{abstract}

MSC: 26D15; 26E70

Keywords: Fractional calculus; Calculus on time scales; Conformable nabla derivative; Conformable nabla integral; Hardy's inequality

\section{Introduction}

Fractional calculus theory has an important role in the mathematical analysis and applications. Fractional calculus (FC), the theory of integrals and derivatives of noninteger order, is a field of research with a history dating back to Abel, Riemann, and Liouville (see [33] for a historical summary). Indeed, the most famous and extensively studied formulation,

$$
I_{a+}^{\alpha} \eta(t)=\frac{1}{\Gamma(\alpha)} \int_{a}^{x}(x-t)^{\alpha-1} \eta(t) d t
$$

is called the Riemann-Liouville fractional integral in their honor. The corresponding fractional derivative is obtained by a composition of fractional integral with integer order derivative.

The definitions of fractional integrals and derivatives are not unique, and many definitions of fractional derivative operators have been introduced and successfully applied to solve complex systems in science and engineering (see [17, 32, 37]). Recently, study on fractional dynamic equations is very widespread around the world and is useful in pure and applied mathematics, physics, engineering, biology, economics, etc. They use an integral in its formulation, especially Cauchy's integral formula with some modifications. Therefore, they sometimes require a difficult calculation to obtain. Riemann-Liouville and Caputo fractional derivatives do not satisfy the nonlinear derivative rules as product,

(c) The Author(s) 2021. This article is licensed under a Creative Commons Attribution 4.0 International License, which permits use sharing, adaptation, distribution and reproduction in any medium or format, as long as you give appropriate credit to the original author(s) and the source, provide a link to the Creative Commons licence, and indicate if changes were made. The images or other third party material in this article are included in the article's Creative Commons licence, unless indicated otherwise in a credit line to the material. If material is not included in the article's Creative Commons licence and your intended use is not permitted by statutory regulation or exceeds the permitted use, you will need to obtain permission directly from the copyright holder. To view a copy of this licence, visit http://creativecommons.org/licenses/by/4.0/. 
quotient, and chain rules. The mean value theorem and Rolle's theorem are not formulated using the definitions of Riemann-Liouville and Caputo fractional derivatives.

Recently, depending just on the basic limit definition of the derivative, Khalil et al. [31] proposed a new simple definition of the fractional derivative called conformable derivative $T_{\alpha} f(t)(\alpha \in(0,1])$ of a function $f: \mathbb{R}^{+} \rightarrow \mathbb{R}$

$$
T_{\alpha} f(t)=\lim _{\epsilon \rightarrow 0} \frac{f\left(t+\epsilon t^{1-\alpha}\right)-f(t)}{\epsilon},
$$

for all $t>0, \alpha \in(0,1]$, this definition found wide resonance in the scientific community interested in fractional calculus, see [29, 30, 48]. Therefore, calculating the derivative by this definition is easily compared to the definitions that are based on integration. The researchers in [31] also suggested a definition for the $\alpha$-conformable integral of a function $\eta$ as follows:

$$
\int_{a}^{b} \eta(t) d_{\alpha} t=\int_{a}^{b} \eta(t) t^{\alpha-1} d t
$$

After that, Abdeljawad [4] made an extensive research of the newly introduced conformable calculus. In his work, he generalized the definition of conformable derivative $T_{\alpha}^{a} f(t)$ for $t>a \in \mathbb{R}^{+}$as follows:

$$
T_{\alpha}^{a} f(t)=\lim _{\epsilon \rightarrow 0} \frac{f\left(t+\epsilon(t-a)^{1-\alpha}\right)-f(t)}{\epsilon}
$$

where $f: \mathbb{R}^{+} \rightarrow \mathbb{R}$. Benkhettou et al. [38] introduced a conformable calculus on an arbitrary time scale, which is a natural extension of the conformable calculus.

In the last few decades many authors pointed out that derivatives and integrals of noninteger order are very suitable for the description of properties of various real materials, e.g., polymers. Fractional derivatives provide an excellent instrument for the description of memory and hereditary properties of various materials and processes. These are some of the advantages of fractional derivatives in comparison with classical integer-order models.

Time scales theory, which has become a trend, began with S. Hilger. In his PhD thesis, this concept was initiated in order to get the continuous theorem and the discrete theorem in one theorem [27]. In [12, 13], Bohner and Peterson introduced the most basic concepts and definitions related to the theory of time scales. Next, some basic definitions and concepts about the fractional analysis, which are used in this manuscript, were given and adapted from [12, 13, 34, 38]. Any nonempty arbitrary closed subset of the real numbers is called a time scale $\mathbb{T}$. We assume that $\mathbb{T}$ has the standard topology on the real numbers $\mathbb{R}$. Now, let $\sigma: \mathbb{T} \rightarrow \mathbb{T}$ be the forward jump operator defined by

$$
\sigma(t):=\inf \{s \in \mathbb{T}: s>t\}, \quad t \in \mathbb{T}
$$

and $\rho: \mathbb{T}: \rightarrow \mathbb{T}$ be the backward jump operator defined by

$$
\rho(t):=\sup \{s \in \mathbb{T}: s<t\}, \quad t \in \mathbb{T} .
$$


In (1.1) and (1.2), we set $\sup \mathbb{T}=\inf \emptyset$ (i.e., $\sigma(t)=t$ if $t$ is the minimum of $\mathbb{T}$ ) and inf $\mathbb{T}=\sup \emptyset$ (i.e., $\rho(t)=t$ if $t$ is the maximum), where $\emptyset$ is the empty set. A point $t \in \mathbb{T}$ with inf $\mathbb{T}<t<$ $\sup \mathbb{T}$ is said to be right-dense if $\sigma(t)=t$, left-dense if $\rho(t)=t$, right-scattered if $\sigma(t)>t$, and left-scattered if $\rho(t)<t$. Points that are simultaneously right-dense and left-dense are called dense points, and points that are simultaneously right-scattered and left-scattered are called isolated points. The forward and backward graininess functions $\mu$ and $\nu$, for a time scale $\mathbb{T}$, are defined by $\mu(t):=\sigma(t)-t$ and $\nu(t):=t-\rho(t)$, respectively.

In [11], the authors studied a version of the nabla conformable fractional derivative on arbitrary time scales. Namely, for a function $\eta: \mathbb{T} \rightarrow \mathbb{R}$, the nabla conformable fractional derivative, $T_{\nabla, \alpha} \eta(t) \in \mathbb{R}$ of order $\alpha \in(0,1]$ at $t \in \mathbb{T}_{\kappa}$ and $t>0$ was defined as: Given any $\epsilon>0$, there is a $\delta$-neighborhood $U_{t} \subset \mathbb{T}$ of $t, \delta>0$ such that

$$
\left|[\eta(\rho(t))-\eta(s)] t^{1-\alpha}-T_{\nabla, \alpha}(\eta)(t)[\rho(t)-s]\right| \leq \varepsilon|\rho(t)-s|
$$

for all $s \in U_{t}$. The nabla conformable fractional integral is defined by

$$
\int \eta(t) \nabla_{\alpha} t=\int \eta(t) t^{\alpha-1} \nabla t
$$

Rahmat et al. [38] presented a new type of conformable nabla derivative and integral which involves the time scale power function $\widehat{G}_{n}(t, s)$ for $s, t \in \mathbb{T}$ and also generalizes the definition of the nabla conformable fractional derivative and integral on time scales in [11]. The time scale power function takes the form $(t-a)^{\eta}$ for $\mathbb{T}=\mathbb{R}$ which reduces to the definition of conformable fractional derivative defined by Khalil et al. [31].

Definition 1.1 Let $[s, t] \subset \mathbb{T}$ and $s<t$. The generalized time scale power function $\widehat{G}_{n}$ : $\mathbb{T} \times \mathbb{T} \longrightarrow \mathbb{R}^{+}$for $n \in \mathbb{N}_{0}$ is defined by

$$
\widehat{G}_{n}(t, s)= \begin{cases}(t-s)^{n}, & \text { if }[t, s] \text { dense; } \\ \prod_{j=0}^{n-1}\left(t-\rho^{j}(s)\right), & \text { if }[t, s] \text { isolated; }\end{cases}
$$

and its inverse function $\widehat{G}_{-n}: \mathbb{T} \times \mathbb{T} \longrightarrow \mathbb{R}^{+}$is then given by

$$
\widehat{G}_{-n}(t, s)= \begin{cases}(t-s)^{-n}, & \text { if }[t, s] \text { dense; } \\ \frac{1}{\prod_{j=0}^{n-1}\left(\rho^{n}(t)-\rho^{j}(s)\right)}, & \text { if }[t, s] \text { isolated }\end{cases}
$$

We use the convention $\widehat{G}_{0}(t, s)=1$ for all $s, t \in \mathbb{T}$.

Corollary 1.2 For $h>0, \mathbb{T}=h \mathbb{Z}=\{h k: k \in \mathbb{Z}\}$, we have $\rho^{k}(s)=s-k h$. Then

$$
\begin{aligned}
\widehat{G}_{n}(t, s) & =(t-s)_{h}^{(n)} \\
& =\prod_{j=0}^{n-1}(t-s+j h) \\
& =h^{n}\left(\frac{t-s}{h}\right)^{(n)}, \quad n \in \mathbb{N},
\end{aligned}
$$


and

$$
\begin{aligned}
\widehat{G}_{n}(t, s) & =(t-s)_{h}^{(-n)} \\
& =\frac{1}{\prod_{j=0}^{n-1}(t-n-s+j h)} \\
& =h^{-n}\left(\frac{t-n-s}{h}+n\right)^{(n)}, \quad n \in \mathbb{N},
\end{aligned}
$$

where

$$
x^{(n)}=\frac{\Gamma(x+n)}{\Gamma(x)} \quad \text { and } \quad x^{(-n)}=\frac{1}{(x-n)^{(n)}}=\frac{\Gamma(x-n)}{\Gamma(x)}, \quad n \in \mathbb{N}, x^{(0)}=1 .
$$

For $\mathbb{T}=q^{\mathbb{N}_{0}}$, we have $\rho^{k}(s)=s q^{-k}$. Then we write

$$
\begin{aligned}
\widehat{G}_{n}(t, s) & =(t-s)_{\tilde{q}}^{(n)} \\
& =\prod_{j=0}^{n-1}\left(t-s q^{-j}\right) \\
& =t^{n} \prod_{j=0}^{n-1}\left(1-\frac{\tilde{q}^{j} s}{t}\right), \quad\left(0<\tilde{q}=\frac{1}{q}<1\right) .
\end{aligned}
$$

Remark 1.3 Regarding the generalization of the power function $\widehat{G}_{\alpha}(t, s)$ to real values of $\alpha \geq 0$ (instead of integers), we recall a broadly accepted extension of its particular cases (1.5) and (1.7) in the form (see [14])

$$
(t-s)_{h}^{(\alpha)}=h^{\alpha} \frac{\Gamma\left(\frac{t-s}{h}+\alpha\right)}{\Gamma\left(\frac{t-s}{h}\right)}, \quad(t-s)_{\tilde{q}}^{(\alpha)}=t^{\alpha} \frac{(s / t, \tilde{q})_{\infty}}{\left(\tilde{q}^{\alpha} s / t, \tilde{q}\right)_{\infty}}, \quad t \neq 0,
$$

where

$$
(p, \tilde{q})_{\infty}=\prod_{j=0}^{\infty}(1-p \tilde{q})
$$

Definition 1.4 (Conformable nabla derivative) Given a function $f: \mathbb{T} \longrightarrow \mathbb{R}$ and $a \in \mathbb{T}$, $f$ is $(\gamma, a)$-nabla differentiable at $t>a$, if it is nabla differentiable at $t$, and its $(\gamma, a)$-nabla derivative is defined by

$$
\nabla_{a}^{\gamma} f(t)=\widehat{G}_{1-\gamma}(t, a) f^{\nabla}(t), \quad t>a,
$$

where the function $\widehat{G}_{1-\gamma}(t, a)$ is as defined in (1.3). If $\nabla_{a}^{\gamma}[f(t)]$ exists in some interval $(a, a+$ $\epsilon)_{\mathbb{T}}, \epsilon>0$, then we define

$$
\nabla_{a}^{\gamma}[f(a)]=\lim _{t \rightarrow a^{+}} \nabla_{a}^{\gamma}[f(t)]
$$

if the $\lim _{t \rightarrow a^{+}} \nabla_{a}^{\gamma}[f(t)]$ exists. Moreover, we call $f$ is $(\gamma, a)$-nabla differentiable on $\mathbb{T}_{k}(a \in$ $\mathbb{T}_{k}$ ) provided $\nabla_{a}^{\gamma}[f(t)]$ exists for all $t \in \mathbb{T}_{k}$. The function $\nabla_{a}^{\gamma}: \mathbb{T}_{k} \rightarrow \mathbb{R}$ is then called $(\gamma, a)$ nabla derivative of $f$ on $\mathbb{T}_{k}$. 
Next, we provide the $(\gamma, a)$-nabla derivatives of sums, products, and quotients of $(\gamma, a)$ nabla differentiable functions.

Theorem 1.5 Assume that $f, g: \mathbb{T} \longrightarrow \mathbb{R}$ are $(\gamma, a)$-nabla differentiable at $t \in \mathbb{T}_{k}, t>a$. Then:

(i) The sum $f+g: \mathbb{T} \longrightarrow \mathbb{R}$ is $(\gamma, a)$-nabla differentiable at $t$ with

$$
\nabla_{a}^{\gamma}(r f+s g)(t)=r \nabla_{a}^{\gamma} f(t)+s \nabla_{a}^{\gamma} g(t)
$$

(ii) For all $k \in \mathbb{R}$, then $k f: \mathbb{T} \longrightarrow \mathbb{R}$ is $(\gamma, a)$-nabla differentiable at $t$ with

$$
\nabla_{a}^{\gamma}(k f)(t)=k \nabla_{a}^{\gamma} f(t)
$$

(iii) The product $f g: \mathbb{T} \longrightarrow \mathbb{R}$ is $(\gamma, a)$-nabla differentiable at $t$ with

$$
\nabla_{a}^{\gamma}(f g)(t)=\left[\nabla_{a}^{\gamma} f(t)\right] g(t)+f^{\rho}(t)\left[\nabla_{a}^{\gamma} g(t)\right]
$$

(iv) If $g(t) g^{\rho}(t) \neq 0$, then $f / g$ is $(\gamma, a)$-nabla differentiable at $t$ with

$$
\nabla_{a}^{\gamma}\left(\frac{f}{g}\right)(t)=\frac{\left[\nabla_{a}^{\gamma} f(t)\right] g(t)-f(t)\left[\nabla_{a}^{\gamma} g(t)\right]}{g(t) g^{\rho}(t)} .
$$

Lemma 1.6 (Chain rule) Let $g \in C_{l d}^{\nabla}(\mathbb{T})$ and assume that $f: \mathbb{R} \rightarrow \mathbb{R}$ is a continuously differentiable function. Then $(f \circ g): \mathbb{T} \rightarrow \mathbb{R}$ is $(\gamma, a)$-nabla differentiable and satisfies

$$
\nabla_{a}^{\gamma}(f \circ g)(t)=\left\{\int_{0}^{1} f^{\prime}\left(g(t)-h v(t) g^{\nabla}(t)\right) d h\right\} \nabla_{a}^{\gamma} g(t)
$$

Lemma 1.7 Let $\gamma \in(0,1]$. Assume that $\xi: \mathbb{T} \rightarrow \mathbb{R}$ is continuous and $(\gamma, a)$-nabla differentiable of order $\gamma$ at $t \in \mathbb{T}_{k}$, where $t>a$ and $\eta: \mathbb{R} \rightarrow \mathbb{R}$ is continuously differentiable. Then there is $c$ in the real interval $[\rho(t), t]$ such that

$$
\nabla_{a}^{\gamma}(\eta \circ \xi)(t)=\eta^{\prime}(\xi(c)) \nabla_{a}^{\gamma}(\xi(t))
$$

Definition $1.8\left(\gamma\right.$-nabla integral from $a$ ) Assume that $0<\gamma \leq 1, a, t_{1}, t_{2} \in \mathbb{T}, a \leq t_{1} \leq$ $t_{2}$, and $f \in C_{l d}(\mathbb{T})$, then we say that $f$ is $(\gamma, a)$-nabla integrable on interval $\left[t_{1}, t_{2}\right]$ if the following integral

$$
\begin{aligned}
\nabla_{a}^{-\gamma} f(t) & =\int_{t_{1}}^{t_{2}} f(\tau) \nabla_{a}^{\gamma} \tau \\
& =\int_{t_{1}}^{t_{2}} f(\tau) \widehat{G}_{\gamma-1}\left(\sigma^{\gamma-1}(\tau), a\right) \nabla \tau
\end{aligned}
$$

exists and is finite.

We need the relations between different types calculus on time scales $\mathbb{T}$ and continuous calculus, discrete calculus, and quantum calculus as follows. Note that: For the case $\mathbb{T}=\mathbb{R}$, 
we have the classical conformable integral as defined in [4], namely

$$
\int_{a}^{t} f(\tau) \nabla_{a}^{\gamma} \tau=\int_{a}^{t} f(\tau)(\tau-a)^{\gamma-1} d \tau
$$

For $\mathbb{T}=h \mathbb{Z}, h>0$, we have a new conformable fractional $h$-sum given by

$$
\int_{a}^{t} f(\tau) \nabla_{a}^{\gamma} \tau=\sum_{\tau \in(a, t]} h f(\tau)\left(\rho^{\gamma-1}(\tau)-a\right)_{h}^{(\gamma-1)} .
$$

For $\mathbb{T}=q^{\mathbb{N}_{0}}$, we have a new conformable fractional $q$-sum given by

$$
\int_{a}^{t} f(\tau) \nabla_{a}^{\gamma} \tau=\sum_{\tau \in(a, t]} \tau(1-\tilde{q}) f(\tau)\left(\rho^{\gamma-1}(\tau)-a\right)_{\tilde{q}}^{(\gamma-1)}
$$

Theorem 1.9 Let $\gamma \in(0,1]$ and $a \in \mathbb{T}$. Then, for any ld-continuous function $f: \mathbb{T} \rightarrow \mathbb{R}$, there exists a function $F: \mathbb{T} \longrightarrow \mathbb{R}$ such that

$$
\nabla_{a}^{\gamma} F(t)=f(t) \quad \text { for all } t \in \mathbb{T}_{k} .
$$

The function $F$ is called a $(\gamma, a)$ - nabla antiderivative of $f$.

Theorem 1.10 Let $\gamma \in(0,1]$. If $a, t_{1} t_{2}, t_{3} \in \mathbb{T}, a \leq t_{1} \leq t_{2} \leq t_{3}, \alpha \in \mathbb{R}$, and $f, g \in C_{l d}(\mathbb{T})$, then

(i) $\int_{t_{2}}^{t_{2}}[f(t)+g(t)] \nabla_{a}^{\gamma} t=\int_{t_{1}}^{t_{2}} f(t) \nabla_{a}^{\gamma} t+\int_{t_{1}}^{t_{2}} g(t) \nabla_{a}^{\gamma} t$.

(ii) $\int_{t_{1}}^{t_{2}} \alpha f(t) \nabla_{a}^{\gamma} t=\alpha \int_{t_{1}}^{t_{2}} f(t) \nabla_{a}^{\gamma} t$.

(iii) $\int_{t_{1}}^{t_{2}} f(t) \nabla_{a}^{\gamma} t=-\int_{t_{2}}^{t_{1}} f(t) \nabla_{a}^{\gamma} t$.

(iv) $\int_{t_{1}}^{t_{3}} f(t) \nabla_{a}^{\gamma} s=\int_{t_{1}}^{t_{2}} f(t) \nabla_{a}^{\gamma} t+\int_{t_{2}}^{t_{3}} f(t) \nabla_{a}^{\gamma} t$.

(v) $\int_{t_{1}}^{t_{1}} f(t) \nabla_{a}^{\gamma} t=0$.

(vi) If $|f(t)| \leq g(t)$ on $\left[t_{1}, t_{2}\right]$, then

$$
\left|\int_{t_{1}}^{t_{2}} f(t) \nabla_{a}^{\gamma} t\right| \leq \int_{t_{1}}^{t_{2}} g(t) \nabla_{a}^{\gamma} t
$$

The study of Hardy-type inequalities attracted and still attracts the attention of many researchers. Over several decades many generalizations, extensions, and refinements have been made to the above inequalities, we refer the interested reader to the papers $[1,8,9$, $18,19,23,24,35,36,41,44]$, see also $[2,21,22,24]$ and the references cited therein.

Hardy [25] established the classical discrete inequality.

Theorem 1.11 Let $\{\varrho(l)\}_{l=1}^{\infty}$ be a sequence of nonnegative real numbers. For $1<p$, we have

$$
\sum_{l=1}^{\infty} \frac{1}{l^{p}}\left(\sum_{J=1}^{l} \varrho(J)\right)^{p} \leq\left(\frac{p}{p-1}\right)^{p} \sum_{\imath=1}^{\infty} \varrho^{p}(\imath) .
$$

In 1925, by using the calculus of variations, Hardy [26] introduced a continuous version of inequality (1.15). 
Theorem 1.12 Suppose that the continuous function $\eta \geq 0$ on $[0, \infty)$. For $1<p$, we have

$$
\int_{0}^{\infty} \frac{1}{\pi^{p}}\left(\int_{0}^{\pi} \eta(s) d s\right)^{p} d \pi \leq\left(\frac{p}{p-1}\right)^{p} \int_{0}^{\infty} \eta^{p}(\pi) d \pi
$$

The constant $\left(\frac{p}{p-1}\right)^{p}$ in (1.16) is sharp.

Copson [16] obtained another classical discrete inequality of Hardy type.

Theorem 1.13 Let $\{\varrho(l)\}_{l=1}^{\infty}$ be a sequence of nonnegative real numbers. For $1<p$, we have

$$
\sum_{i=1}^{\infty}\left(\sum_{J=l}^{\infty} \varrho(J)\right)^{p} \leq p^{p} \sum_{\imath=1}^{\infty}(\iota \varrho(l))^{p} .
$$

Renaud [40] proved the following two results which are the reverse discrete and continuous versions of inequality (1.17).

Theorem 1.14 Suppose that $\{\varrho(l)\}_{l=1}^{\infty}$ is a sequence of nonnegative and nonincreasing real numbers. For $1<p$, we have

$$
\sum_{l=1}^{\infty}\left(\sum_{J=l}^{\infty} \varrho(J)\right)^{p} \geq \sum_{l=1}^{\infty} l^{p} \varrho^{p}(\imath) .
$$

Theorem 1.15 Suppose that $\eta$ is a nonnegative and nonincreasing function on the interval $[0, \infty)$. For $1<p$, we have

$$
\int_{0}^{\infty}\left(\int_{\pi}^{\infty} \eta(s) d s\right)^{p} d \pi \geq \int_{0}^{\infty} \pi^{p} \eta^{p}(\pi) d \pi
$$

Renaud [40] proved the following result.

Theorem 1.16 Suppose that $\eta$ is a nonnegative and nonincreasing function on the interval $[0, \infty)$. For $1<p$, we have

$$
\int_{0}^{\infty} \frac{1}{\pi^{p}}\left(\int_{0}^{\pi} \eta(s) d s\right)^{p} d \pi \geq \frac{p}{p-1} \int_{0}^{\infty} \eta^{p}(\pi) d \pi
$$

In $[3,6,12,19,20,24,28,39]$ many mathematicians have investigated several new forms of dynamic inequalities. In 2005, ̌̌ehák [39] was the first mathematician that introduced the time scales version of Hardy inequality which unifies inequalities (1.15) and (1.16) as follows.

Theorem 1.17 Let $\mathbb{T}$ be a time scale, and $f \in C_{r d}\left([a, \infty)_{\mathbb{T}},[0, \infty)\right)$. If $p>1$, then

$$
\int_{a}^{\infty}\left(\frac{\int_{a}^{\sigma(t)} \eta(s) \Delta s}{\sigma(t)-a}\right)^{p} \Delta t<\left(\frac{p}{p-1}\right)^{p} \int_{a}^{\infty} \eta^{p}(t) \Delta t
$$

unless $\eta \equiv 0$. 
Furthermore, if $\mu(t) / t \rightarrow 0$ as $t \rightarrow \infty$, then inequality (1.21) is sharp.

Saker et al. [43] studied the following results on time scale.

Theorem 1.18 Let $\mathbb{T}$ be a time scale and $1 \leq c \leq k$. Let

$$
\chi(t)=\int_{a}^{t} \lambda(s) \Delta s \quad \text { for any } t \in[a, \infty)_{\mathbb{T}},
$$

and define

$$
\Theta(t)=\int_{a}^{t} \lambda(s) \xi(s) \Delta s \quad \text { for any } t \in[a, \infty)_{\mathbb{T}} .
$$

Then

$$
\int_{a}^{\infty} \frac{\lambda(t)}{\left(\chi^{\sigma}(t)\right)^{c}}\left(\Theta^{\sigma}(t)\right)^{k} \Delta t \leq \frac{k}{c-1} \int_{a}^{\infty} \chi^{1-c}(t) \lambda(t) \xi(t)(\Theta(t))^{k-1} \Delta t .
$$

and

$$
\int_{a}^{\infty} \frac{\lambda(t)}{\left(\chi^{\sigma}(t)\right)^{c}}\left(\Theta^{\sigma}(t)\right)^{k} \Delta t \leq\left(\frac{k}{c-1}\right)^{k} \int_{a}^{\infty} \frac{\left(\chi^{\sigma}(t)\right)^{(k-1) c}}{(\chi(t))^{k(c-1)}} \lambda(t) \xi^{k}(t) \Delta t .
$$

Theorem 1.19 Let $\mathbb{T}$ be a time scale and $k>1$ and $0 \leq c<1$. Let $\chi$ be defined as in (1.22) and define

$$
\bar{\Theta}(t)=\int_{t}^{\infty} \lambda(s) \xi(s) \Delta s \quad \text { for any } t \in[a, \infty)_{\mathbb{T}} .
$$

Then

$$
\int_{a}^{\infty} \frac{\lambda(t)}{\left(\chi^{\sigma}(t)\right)^{c}}(\bar{\Theta}(t))^{k} \Delta t \leq \frac{k}{1-c} \int_{a}^{\infty}\left(\chi^{\sigma}(t)\right)^{1-c} \lambda(t) \xi(t)(\bar{\Theta}(t))^{k-1} \Delta t
$$

and

$$
\int_{a}^{\infty} \frac{\lambda(t)}{\left(\chi^{\sigma}(t)\right)^{c}}(\bar{\Theta}(t))^{k} \Delta t \leq\left(\frac{k}{1-c}\right)^{k} \int_{a}^{\infty}\left(\chi^{\sigma}(t)\right)^{k-c} \lambda(t) \xi^{k}(t) \Delta t
$$

Agarwal et al. [7] generalized inequality (1.20) on time scales as follows: for $p>1$,

$$
\int_{0}^{\infty} \frac{1}{t^{p}}\left(\int_{0}^{t} \eta(s) \Delta s\right)^{p} \Delta t \geq \frac{p}{p-1} \int_{0}^{\infty} \eta^{p}(t) \Delta t
$$

In 2020, El-Deeb et al. [23] established a generalization of (1.24) which unify (1.18) and (1.19) in the following form: for $1 \leq p$ and $1<\gamma$, we obtain

$$
\int_{a}^{\infty} \frac{\tilde{\lambda}(\zeta) \breve{\Psi}^{p}(\zeta)}{\tilde{\Lambda} \hat{\gamma}(\zeta)} \Delta \zeta \geq \frac{p}{\hat{\gamma}-1} \int_{a}^{\infty} \tilde{\lambda}(\zeta) \tilde{\Lambda}^{p-\hat{\gamma}}(\zeta) \eta^{p}(\zeta) \Delta \zeta
$$


where

$$
\breve{\Psi}(\zeta)=\int_{a}^{\zeta} \tilde{\lambda}(\eta) \eta(s) \Delta \eta \quad \text { and } \quad \tilde{\Lambda}(\zeta)=\int_{a}^{\zeta} \tilde{\lambda}(\eta) \Delta \eta
$$

For more results on the Hardy-type inequalities on time scales, we refer the interested reader to $[8,18,35,36,44]$.

A few years ago, by using conformable calculus, a lot of papers have been published for conformable inequalities, and several authors have investigated different inequalities like Hardy's inequality [42, 49], Hermite-Hadamard's inequality [5, 15, 47], Opial's inequality [10, 45], and Steffensen's inequality [46]. For example, in 2020, Saker et al. [42] gave $\alpha$ conformable versions of Theorem 1.18 and Theorem 1.19 on time scales as follows.

Theorem 1.20 Let $\mathbb{T}$ be a time scale with $1 \leq c \leq k$, and define

$$
\chi(x)=\int_{a}^{x} \lambda(s) \Delta_{\alpha} s \text { and } \Theta(x)=\int_{a}^{x} \lambda(s) \xi(s) \Delta_{\alpha} s .
$$

If

$$
\Theta(\infty)<\infty \text { and } \int_{a}^{\infty} \frac{\lambda(s)}{\left(\chi^{\sigma}(s)\right)^{c-\alpha+1}} \Delta_{\alpha} s<\infty,
$$

then

$$
\int_{a}^{\infty} \frac{\lambda(x)}{\left(\chi^{\sigma}(x)\right)^{c-\alpha+1}}(\Theta(x))^{k} \Delta_{\alpha} x \leq\left(\frac{k}{c-\alpha}\right)^{k} \int_{a}^{\infty} \frac{\lambda(x)(\chi(x))^{K(\alpha-c)}}{\left(\chi^{\sigma}(x)\right)^{(1-k)(c-\alpha+1)}} \xi^{k}(x) \Delta_{\alpha} x .
$$

Very recently, Zakarya et al. [49] gave an $\alpha$-conformable version of Theorem 1.20 on time scales as follows.

Theorem 1.21 Assume that $\mathbb{T}$ is a time scale with $\omega \in(0, \infty)_{\mathbb{T}}$. If $k \leq 0<h<1$ and $\alpha \in$ $(0,1]$, define

$$
\chi(t)=\int_{t}^{\infty} \lambda(s) \Delta_{\alpha} s \quad \text { and } \quad \Theta(t)=\int_{\omega}^{t} \lambda(s) \xi(s) \Delta_{\alpha} s
$$

Then

$$
\int_{\omega}^{\infty} \frac{\lambda(t)}{\chi^{k-\alpha+1}(t)}\left(\Theta^{\sigma}(t)\right)^{h} \Delta_{\alpha} t \geq\left(\frac{h}{\alpha-k}\right)^{h} \int_{\omega}^{\infty} \lambda(t) \xi^{h}(t) \chi^{h-k+\alpha-1}(t) \Delta_{\alpha} t .
$$

In this paper, motivated by the results in $[7,23,43]$, we introduce a new nabla version of Hardy-type dynamic inequalities via conformable fractional $\nabla$-integral of order $\gamma \in(0,1]$ on time scales. These inequalities have a completely new form. Therefore, as special case, we obtain some new conformable fractional $h$-sum inequalities, new conformable fractional $q$-sum inequalities, and new classical conformable fractional integral inequalities. 


\section{Main results}

Now, we are ready to state and prove our main results. Throughout this section, any time scale $\mathbb{T}$ is unbounded above, and we will assume that the right-hand sides of the inequalities converge if the left-hand sides converge.

Theorem 2.1 Assume that $\mathbb{T}$ is a time scale with $0 \leq r \in \mathbb{T}$. Moreover, suppose that $f$ and $\lambda$ are nonnegative Id-continuous functions on $[r, \infty)_{\mathbb{T}}$ with $f$ nondecreasing. If $p \geq 1$ and $\beta \leq 0$, then for $t \geq a \in \mathbb{T}$ and $\gamma \in(0,1]$ we have that

$$
\int_{r}^{\infty} \frac{\lambda(t) \Phi^{p-\gamma+1}(t)}{\left(\Lambda^{\rho}(t)\right)^{\beta}} \nabla_{a}^{\gamma} t \leq \int_{r}^{\infty} \frac{\lambda(t) \Lambda^{p-\gamma+1}(t) f^{p-\gamma+1}(t)}{\left(\Lambda^{\rho}(t)\right)^{\beta}} \nabla_{a}^{\gamma} t,
$$

where

$$
\Phi(t)=\int_{t}^{\infty} \lambda(s) f(s) \nabla_{a}^{\gamma} s \quad \text { and } \quad \Lambda(t)=\int_{r}^{t} \lambda(s) \nabla_{a}^{\gamma} s
$$

Proof As $f$ is nondecreasing, we have for $x \geq t \geq r$

$$
F(x, t)=\int_{t}^{x} \lambda(s) f(s) \nabla_{a}^{\gamma} s \leq f(x) \int_{t}^{x} \lambda(s) \nabla_{a}^{\gamma} s .
$$

Then

$$
f(x) F^{p-\gamma}(x, t) \leq\left[\int_{t}^{x} \lambda(s) \nabla_{a}^{\gamma} s\right]^{p-\gamma} f^{p-\gamma+1}(x) .
$$

Applying the chain rule (1.9) and using $\nabla_{a}^{\gamma, x} F(x, t)=\lambda(x) f(x) \geq 0$, where $\nabla_{a}^{\gamma, x}$ denotes the $(\gamma, a)$-nabla derivative with respect to $x$, we get

$$
\begin{aligned}
\nabla_{a}^{\gamma, x}\left(F^{p-\gamma+1}(x, t)\right) & =(p-\gamma+1) \nabla_{a}^{\gamma, x} F(x, t) \int_{0}^{1}[(1-h) F(x, t)+h F(\rho(x), t)]^{p-\gamma} d h \\
& \leq(p-\gamma+1) \lambda(x) f(x) \int_{0}^{1}[(1-h) F(x, t)+h F(x, t)]^{p-\gamma} d h \\
& =(p-\gamma+1) \lambda(x) f(x) F^{p-\gamma}(x, t) .
\end{aligned}
$$

Combining (2.2) with (2.3) gives

$$
\nabla_{a}^{\gamma, x}\left(F^{p-\gamma+1}(x, t)\right) \leq(p-\gamma+1) \lambda(x)\left[\int_{t}^{x} \lambda(s) \nabla_{a}^{\gamma} s\right]^{p-\gamma} f^{p-\gamma+1}(x)
$$

and so (note that $x \geq t \geq r$ and hence, because $\Lambda$ is nondecreasing, $\left(0 \leq\left(\Lambda^{\rho}(x)\right)^{\beta} \leq\right.$ $\left.\left.\Lambda^{\rho}(t)\right)^{\beta}\right)$

$$
\begin{aligned}
\frac{\lambda(t) \nabla_{a}^{\gamma, x}\left(F^{p-\gamma+1}(x, t)\right)}{\left(\Lambda^{\rho}(t)\right)^{\beta}} & \leq \frac{(p-\gamma+1) \lambda(t) \lambda(x)}{\left(\Lambda^{\rho}(t)\right)^{\beta}}\left[\int_{t}^{x} \lambda(s) \nabla_{a}^{\gamma} s\right]^{p-\gamma} f^{p-\gamma+1}(x) \\
& \leq \frac{(p-\gamma+1) \lambda(t) \lambda(x)}{\left(\Lambda^{\rho}(x)\right)^{\beta}}\left[\int_{t}^{x} \lambda(s) \nabla_{a}^{\gamma} s\right]^{p-\gamma} f^{p-\gamma+1}(x) .
\end{aligned}
$$


Integrating both sides with respect to $x$ over $[t, \infty)_{\mathbb{T}}$ gives

$$
\begin{aligned}
\frac{\lambda(t) \Phi^{p-\gamma+1}(t)}{\left(\Lambda^{\rho}(t)\right)^{\beta}} & =\int_{t}^{\infty} \frac{\lambda(t) \nabla_{a}^{\gamma, x}\left(F^{p-\gamma+1}(x, t)\right)}{\left(\Lambda^{\rho}(t)\right)^{\gamma}} \nabla_{a}^{\gamma} x \\
& \leq(p-\gamma+1) \int_{t}^{\infty} \frac{\lambda(t) \lambda(x)}{\left(\Lambda^{\rho}(x)\right)^{\beta}}\left[\int_{t}^{x} \lambda(s) \nabla_{a}^{\gamma, x} s\right]^{p-\gamma} f^{p-\gamma+1}(x) \nabla_{a}^{\gamma} x .
\end{aligned}
$$

Integrating both sides again, but this time with respect to $t$ over $[r, \infty)_{\mathbb{T}}$, produces

$$
\begin{aligned}
& \int_{r}^{\infty} \frac{\lambda(t) \Phi^{p-\gamma+1}(t)}{\left(\Lambda^{\rho}(t)\right)^{\beta}} \nabla_{a}^{\gamma} t \\
& \quad \leq(p-\gamma+1) \int_{r}^{\infty}\left[\int_{t}^{\infty} \frac{\lambda(t) \lambda(x)}{\left(\Lambda^{\rho}(x)\right)^{\beta}}\left[\int_{t}^{x} \lambda(s) \nabla_{a}^{\gamma} s\right]^{p-\gamma} f^{p-\gamma+1}(x) \nabla_{a}^{\gamma} x\right] \nabla_{a}^{\gamma} t .
\end{aligned}
$$

Using Fubini's theorem on time scales, inequality (2.4) can be rewritten as

$$
\begin{aligned}
& \int_{r}^{\infty} \frac{\lambda(t) \Phi^{p-\gamma+1}(t)}{\left(\Lambda^{\rho}(t)\right)^{\beta}} \nabla_{a}^{\gamma} t \\
& \quad \leq(p-\gamma+1) \\
& \quad \times \int_{r}^{\infty} \lambda(x)\left(\Lambda^{\rho}(x)\right)^{-\beta} f^{p-\gamma+1}(x)\left[\int_{r}^{x} \lambda(t)\left[\int_{t}^{x} \lambda(s) \nabla_{a}^{\gamma} s\right]^{p-\gamma} \nabla_{a}^{\gamma} t\right] \nabla_{a}^{\gamma} x .
\end{aligned}
$$

Now, from the chain rule (1.10), there exists $c \in[\rho(t), t]$ such that (here $\nabla_{a}^{\gamma, t}$ denotes the $(\gamma, a)$-nabla derivative with respect to $t)$

$$
\begin{aligned}
\nabla_{a}^{\gamma, t}\left[-\left(\int_{t}^{x} \lambda(s) \nabla_{a}^{\gamma}\right)^{p-\gamma+1}\right] & =(p-\gamma+1) \lambda(t)\left[\int_{c}^{x} \lambda(s) \nabla_{a}^{\gamma} s\right]^{p-\gamma} \\
& \geq(p-\gamma+1) \lambda(t)\left[\int_{t}^{x} \lambda(s) \nabla_{a}^{\gamma} s\right]^{p-\gamma} .
\end{aligned}
$$

Substituting (2.6) into (2.5) leads to

$$
\begin{aligned}
& \int_{r}^{\infty} \frac{\lambda(t) \Phi^{p-\gamma+1}(t)}{\left(\Lambda^{\rho}(t)\right)^{\beta}} \nabla_{a}^{\gamma} t \\
& \quad \leq \int_{r}^{\infty} \lambda(x)\left(\Lambda^{\rho}(x)\right)^{-\beta} f^{p-\gamma+1}(x)\left[\int_{r}^{x}-\nabla_{a}^{\gamma, t}\left[\left(\int_{t}^{x} \lambda(s) \nabla_{a}^{\gamma} s\right)^{p-\gamma+1}\right] \nabla_{a}^{\gamma} t\right] \nabla_{a}^{\gamma} x \\
& \quad=\int_{r}^{\infty} \frac{\lambda(x) \Lambda^{p-\gamma+1}(x) f^{p-\gamma+1}(x)}{\left(\Lambda^{\rho}(x)\right)^{\beta}} \nabla_{a}^{\gamma} x .
\end{aligned}
$$

This shows the validity of inequality (2.1).

Now, as special cases of our results, we give the continuous, discrete, and quantum $\alpha$ conformable inequalities. Namely, in cases of time scales $\mathbb{T}=\mathbb{R}, \mathbb{T}=h \mathbb{Z}, \mathbb{T}=\mathbb{Z}$, and $\mathbb{T}=$ $q^{\mathbb{N}_{0}}$.

Corollary 2.2 If $\mathbb{T}=\mathbb{R}$ in Theorem 2.1, inequality (2.1) reduces to

$$
\int_{r}^{\infty} \frac{\lambda(t) \Phi^{p-\gamma+1}(t)}{\Lambda^{\beta}(t)}(t-a)^{\gamma-1} d t \leq \int_{r}^{\infty} \lambda(t) \Lambda^{p-\beta}(t) f^{p-\gamma+1}(t)(t-a)^{\gamma-1} d t
$$


where

$$
\Phi(t)=\int_{t}^{\infty} \lambda(s) f(s)(s-a)^{\gamma-1} d s \text { and } \quad \Lambda(t)=\int_{r}^{t} \lambda(s)(s-a)^{\gamma-1} d s .
$$

Corollary 2.3 If $\mathbb{T}=h \mathbb{Z}$ in Theorem 2.1, inequality (2.1) reduces to

$$
\begin{aligned}
& \sum_{t=\frac{r}{h}}^{\infty} \frac{\lambda(h t) \Phi^{p-\gamma+1}(h t)}{\Lambda^{\beta}(h t-h)}\left(\rho^{\gamma-1}(h t)-a\right)_{h}^{(\gamma-1)} \\
& \quad \leq \sum_{t=\frac{r}{h}}^{\infty} \frac{\lambda(h t) \Lambda^{p-\gamma+1}(h t) f^{p-\gamma+1}(h t)}{\Lambda^{\beta}(h t-h)}\left(\rho^{\gamma-1}(h t)-a\right)_{h}^{(\gamma-1)},
\end{aligned}
$$

where

$$
\begin{aligned}
& \Phi(t)=h \sum_{s=\frac{t}{h}}^{\infty} \lambda(h s) f(h s)\left(\rho^{\gamma-1}(h s)-a\right)_{h}^{(\gamma-1)} \text { and } \\
& \Lambda(t)=h \sum_{s=\frac{r}{h}}^{\frac{t}{h}-1} \lambda(h s)\left(\rho^{\gamma-1}(h s)-a\right)_{h}^{(\gamma-1)} .
\end{aligned}
$$

Corollary 2.4 For $\mathbb{T}=\mathbb{Z}$, we simply take $h=1$ in Corollary 2.3. In this case, inequality (2.1) reduces to

$$
\sum_{t=r}^{\infty} \frac{\lambda(t) \Phi^{p-\gamma+1}(t)}{\Lambda^{\beta}(t-1)}\left(\rho^{\gamma-1}(t)-a\right)^{(\gamma-1)} \leq \sum_{t=r}^{\infty} \frac{\lambda(t) \Lambda^{p-\gamma+1}(t) f^{p-\gamma+1}(t)}{\Lambda^{\beta}(t-1)}\left(\rho^{\gamma-1}(t)-a\right)^{(\gamma-1)}
$$

where

$$
\Phi(t)=\sum_{s=t}^{\infty} \lambda(s) f(s)\left(\rho^{\gamma-1}(s)-a\right)^{(\gamma-1)} \quad \text { and } \quad \Lambda(t)=\sum_{s=r}^{t-1} \lambda(s)\left(\rho^{\gamma-1}(t)-a\right)^{(\gamma-1)} .
$$

Corollary 2.5 If $\mathbb{T}=q^{\mathbb{N} 0}$ in Theorem 2.1, inequality (2.1) reduces to

$$
\begin{aligned}
& \sum_{t \in(r, \infty)} \frac{t\left(\rho^{\gamma-1}(t)-a\right)_{\tilde{q}}^{(\gamma-1)} \lambda(t) \Phi^{p-\gamma+1}(t)}{\Lambda^{\beta}(\rho(t))} \\
& \quad \leq \sum_{t \in(r, \infty)} \frac{t\left(\rho^{\gamma-1}(t)-a\right)_{\tilde{q}}^{(\gamma-1)} \lambda(t) \Lambda^{p-\gamma+1}(t) f^{p-\gamma+1}(t)}{\Lambda^{\beta}(\rho(t))},
\end{aligned}
$$

where

$$
\begin{aligned}
& \Phi(t)=(\tilde{q}-1) \sum_{s \in(t, \infty)} s \lambda(s) f(s)\left(\rho^{\gamma-1}(s)-a\right)_{\tilde{q}}^{(\gamma-1)} \text { and } \\
& \Lambda(t)=(\tilde{q}-1) \sum_{s \in(r, t)} s \lambda(s)\left(\rho^{\gamma-1}(s)-a\right)_{\tilde{q}}^{(\gamma-1)} .
\end{aligned}
$$


It is interesting to discuss inequality (2.1) after changing the limit of integral $\int_{r}^{t} \lambda(s) \nabla_{a}^{\gamma} s$ to be from $t$ to $\infty$. Let us do that in the following theorem.

Theorem 2.6 Under the same hypotheses of Theorem 2.1 with $\beta>1$, then we have that

$$
\int_{r}^{\infty} \frac{\lambda(t) \Phi^{p-\gamma+1}(t)}{\Omega^{\beta-\gamma+1}(t)} \nabla_{a}^{\gamma} t \leq \frac{p-\gamma+1}{\beta-\gamma} \int_{r}^{\infty} \lambda(t) \Omega^{p-\beta}(t) f^{p-\gamma+1}(t) \nabla_{a}^{\gamma} t,
$$

where

$$
\Phi(r)=0, \quad \Phi(t)=\int_{t}^{\infty} \lambda(s) f(s) \nabla_{a}^{\gamma} s \quad \text { and } \quad \Omega(t)=\int_{t}^{\infty} \lambda(s) \nabla_{a}^{\gamma} s
$$

Proof Since $f$ is nondecreasing, we have for $t \geq x \geq r$

$$
\Phi(x)=\int_{x}^{\infty} \lambda(s) f(s) \nabla_{a}^{\gamma} s \geq f(x) \Omega(x) .
$$

So,

$$
f(x) \Phi^{p-\gamma}(x) \geq \Omega^{p-\gamma}(x) f^{p-\gamma+1}(x) .
$$

By utilizing the chain rule (1.9) and using $\nabla_{a}^{\gamma}(\Phi(x))=-\lambda(x) f(x) \leq 0$, we get

$$
\begin{aligned}
\nabla_{a}^{\gamma}\left(\Phi^{p-\gamma+1}(x)\right) & =(p-\gamma+1) \nabla_{a}^{\gamma}(\Phi(x)) \int_{0}^{1}[(1-h) \Phi(x)+h \Phi(\rho(x))]^{p-\gamma} d h \\
& \leq-(p-\gamma+1) \lambda(x) f(x) \int_{0}^{1}[h \Phi(x)+(1-h) \Phi(x)]^{p-\gamma} d h \\
& =-(p-\gamma+1) \lambda(x) f(x) \Phi^{p-\gamma}(x) .
\end{aligned}
$$

From (2.8) and (2.9) we get

$$
\nabla_{a}^{\gamma}\left(\Phi^{p-\gamma+1}(x)\right) \leq-(p-\gamma+1) \lambda(x) \Omega^{p-\gamma}(x) f^{p-\gamma+1}(x)
$$

Thus,

$$
\frac{\lambda(t) \nabla_{a}^{\gamma}\left(\Phi^{p-\gamma+1}(x)\right)}{\Omega^{\beta-\gamma+1}(t)} \leq \frac{-(p-\gamma+1) \lambda(t) \lambda(x) \Omega^{p-\gamma}(x) f^{p-\gamma+1}(x)}{\Omega^{\beta-\gamma+1}(t)} .
$$

Therefore, upon integrating both sides with respect to $x$ over $[r, t]_{\mathbb{T}}$,

$$
\begin{aligned}
\frac{\lambda(t)\left[\Phi^{p-\gamma+1}(t)-\Phi^{p-\gamma+1}(r)\right]}{\Omega^{\beta-\gamma+1}(t)} & =\int_{r}^{t} \frac{\lambda(t) \nabla_{a}^{\gamma}\left(\Phi^{p-\gamma+1}(x)\right)}{\Omega^{\beta-\gamma+1}(t)} \nabla_{a}^{\gamma} x \\
& \leq-(p-\gamma+1) \int_{r}^{t} \frac{\lambda(t) \lambda(x) \Omega^{p-\gamma}(x) f^{p-\gamma+1}(x)}{\Omega^{\beta-\gamma+1}(t)} \nabla_{a}^{\gamma} x .
\end{aligned}
$$

Since $\Phi^{p-\gamma+1}(r)=0$, we have

$$
\frac{\lambda(t) \Phi^{p-\gamma+1}(t)}{\Omega^{\beta-\gamma+1}(t)} \leq-(p-\gamma+1) \int_{r}^{t} \frac{\lambda(t) \lambda(x) \Omega^{p-\gamma}(x) f^{p-\gamma+1}(x)}{\Omega^{\beta-\gamma+1}(t)} \nabla_{a}^{\gamma} x .
$$


Then, by integrating both sides with respect to $t$ over $[r, \infty)_{\mathbb{T}}$, we get

$$
\begin{aligned}
& \int_{r}^{\infty} \frac{\lambda(t) \Phi^{p-\gamma+1}(t)}{\Omega^{\beta-\gamma+1}(t)} \nabla_{a}^{\gamma} t \\
& \quad \leq-(p-\gamma+1) \int_{r}^{\infty}\left[\int_{r}^{t} \frac{\lambda(t) \lambda(x) \Omega^{p-\gamma}(x) f^{p-\gamma+1}(x)}{\Omega^{\beta-\gamma+1}(t)} \nabla_{a}^{\gamma} x\right] \nabla_{a}^{\gamma} t .
\end{aligned}
$$

With the help of Fubini's theorem on time scales, inequality (2.10) can be rewritten as

$$
\begin{aligned}
& \int_{r}^{\infty} \frac{\lambda(t) \Phi^{p-\gamma+1}(t)}{\Omega^{\beta-\gamma+1}(t)} \nabla_{a}^{\gamma} t \\
& \quad \leq(p-\gamma+1) \int_{r}^{\infty} \lambda(x) \Omega^{p-\gamma}(x) f^{p-\gamma+1}(x)\left[\int_{x}^{\infty}-\lambda(t) \Omega^{-(\beta-\gamma+1)}(t) \nabla_{a}^{\gamma} t\right] \nabla_{a}^{\gamma} x .
\end{aligned}
$$

From chain rule (1.10), there exists $d \in[\rho(t), t]$ with

$$
\begin{aligned}
-\nabla_{a}^{\gamma}\left(\Omega^{(-\beta+\gamma)}(t)\right) & =-(\beta-\gamma) \lambda(t) \Omega^{-\beta+\gamma-1}(d) \\
& \geq-(\beta-\gamma) \lambda(t) \Omega^{-\beta+\gamma-1}(t) .
\end{aligned}
$$

Combining (2.12) and (2.11) yields

$$
\begin{aligned}
\int_{r}^{\infty} & \frac{\lambda(t) \Phi^{p-\gamma+1}(t)}{\Omega^{\beta-\gamma+1}(t)} \nabla_{a}^{\gamma} t \\
& \leq \frac{p-\gamma+1}{\beta-\gamma} \int_{r}^{\infty} \lambda(x) \Omega^{p-\gamma}(x) f^{p-\gamma+1}(x)\left[\int_{x}^{\infty}-\nabla_{a}^{\gamma}\left(\Omega^{-\beta+\gamma}(t)\right) \nabla_{a}^{\gamma} t\right] \nabla_{a}^{\gamma} x \\
& =\frac{p-\gamma+1}{\beta-\gamma} \int_{r}^{\infty} \lambda(x) \Omega^{p-\beta}(x) f^{p-\gamma+1}(x) \nabla_{a}^{\gamma} x
\end{aligned}
$$

from which inequality (2.7) follows.

We are ready to present several special cases of our results to continuous, discrete, and quantum $\alpha$-conformable inequalities. Namely, in cases of time scales $\mathbb{T}=\mathbb{R}, \mathbb{T}=h \mathbb{Z}, \mathbb{T}=\mathbb{Z}$, and $\mathbb{T}=q^{\mathbb{N}_{0}}$.

Corollary 2.7 If $\mathbb{T}=\mathbb{R}$ in Theorem 2.6, then inequality (2.7) boils down to

$$
\int_{r}^{\infty} \frac{\lambda(t) \Phi^{p-\gamma+1}(t)}{\Omega^{\beta-\gamma+1}(t)}(t-a)^{\gamma-1} d t \leq \frac{p-\gamma+1}{\beta-\gamma} \int_{r}^{\infty} \lambda(t) \Omega^{p-\beta}(t) f^{p-\gamma+1}(t)(t-a)^{\gamma-1} d t
$$

where

$$
\Phi(t)=\int_{t}^{\infty} \lambda(s) f(s)(s-a)^{\gamma-1} d s \quad \text { and } \quad \Omega(t)=\int_{t}^{\infty} \lambda(s)(s-a)^{\gamma-1} d s .
$$

Corollary 2.8 If $\mathbb{T}=h \mathbb{Z}$ in Theorem 2.6, then inequality (2.7) boils down to

$$
\sum_{t=\frac{r}{h}}^{\infty} \frac{\lambda(h t) \Phi^{p-\gamma+1}(h t)}{\Omega^{\beta-\gamma+1}(h t)}\left(\rho^{\gamma-1}(h t)-a\right)_{h}^{(\gamma-1)}
$$




$$
\leq \frac{p-\gamma+1}{\beta-\gamma} \sum_{t=\frac{r}{h}}^{\infty} \lambda(h t) \Omega^{p-\gamma}(h t) f^{p-\gamma+1}(h t)\left(\rho^{\gamma-1}(h t)-a\right)_{h}^{(\gamma-1)},
$$

where

$$
\begin{aligned}
& \Phi(t)=h \sum_{s=\frac{t}{h}}^{\infty} \lambda(h s) f(h s)\left(\rho^{\gamma-1}(h s)-a\right)_{h}^{(\gamma-1)} \text { and } \\
& \Omega(t)=h \sum_{s=\frac{t}{h}}^{\infty} \lambda(h s)\left(\rho^{\gamma-1}(h s)-a\right)_{h}^{(\gamma-1)} .
\end{aligned}
$$

Corollary 2.9 For $\mathbb{T}=\mathbb{Z}$, we simply take $h=1$ in Corollary 2.8. In this case, inequality (2.7) boils down to

$$
\begin{aligned}
& \sum_{t=r}^{\infty} \frac{\lambda(t) \Phi^{p-\gamma+1}(t)}{\Omega^{\beta-\gamma+1}(t)}\left(\rho^{\gamma-1}(t)-a\right)^{(\gamma-1)} \\
& \quad \leq \frac{p-\gamma+1}{\beta-\gamma} \sum_{t=r}^{\infty} \lambda(t) \Omega^{p-\beta}(t) f^{p-\gamma+1}(t)\left(\rho^{\gamma-1}(t)-a\right)^{(\gamma-1)},
\end{aligned}
$$

where

$$
\Phi(t)=\sum_{s=t}^{\infty} \lambda(s) f(s)\left(\rho^{\gamma-1}(s)-a\right)^{(\gamma-1)} \text { and } \Omega(t)=\sum_{s=t}^{\infty} \lambda(s)\left(\rho^{\gamma-1}(s)-a\right)^{(\gamma-1)} .
$$

Corollary 2.10 If $\mathbb{T}=q^{\mathbb{N}_{0}}$ in Theorem 2.6, then inequality (2.7) boils down to

$$
\begin{aligned}
& \sum_{t \in(r, \infty)} \frac{t \lambda(t) \Phi^{p-\gamma+1}(t)}{\Omega^{\beta-\gamma+1}(t)}\left(\rho^{\gamma-1}(t)-a\right)_{\tilde{q}}^{(\gamma-1)} \\
& \quad \leq \frac{p-\gamma+1}{\beta-\gamma} \sum_{t \in(r, \infty)} t \lambda(t) \Omega^{p-\beta}(t) f^{p-\gamma+1}(t)\left(\rho^{\gamma-1}(t)-a\right)_{\tilde{q}}^{(\gamma-1)}
\end{aligned}
$$

where

$$
\begin{aligned}
& \Phi(t)=(\tilde{q}-1) \sum_{s \in(t, \infty)} s \lambda(s) f(s)\left(\rho^{\gamma-1}(t)-a\right)_{\tilde{q}}^{(\gamma-1)} \text { and } \\
& \Omega(t)=(\tilde{q}-1) \sum_{s \in(t, \infty)} s \lambda(s)\left(\rho^{\gamma-1}(t)-a\right)_{\tilde{q}}^{(\gamma-1)}
\end{aligned}
$$

Theorem 2.11 Under the same hypotheses of Theorem 2.1 with $0 \leq \beta \leq 1$, we have that

$$
\int_{r}^{\infty} \frac{\lambda(t) \Psi^{p-\gamma+1}(t)}{\Lambda^{\beta}(t)} \nabla_{a}^{\gamma} t \leq \frac{p-\gamma+1}{\beta-1} \int_{r}^{\infty} \lambda(t) \Lambda^{p-\beta-\gamma+1}(t) f^{p-\gamma+1}(t) \nabla_{a}^{\gamma} t,
$$

where

$$
\Psi(t)=\int_{r}^{t} \lambda(s) f(s) \nabla_{a}^{\gamma} s \quad \text { and } \quad \Lambda(t)=\int_{r}^{t} \lambda(s) \nabla_{a}^{\gamma} s .
$$


Proof As $f$ is nondecreasing, we have for $x \geq r$

$$
\Psi(x)=\int_{r}^{x} \lambda(s) f(s) \nabla_{a}^{\gamma} s \leq f(x) \int_{r}^{x} \lambda(s) \nabla_{a}^{\gamma} s=f(x) \Lambda(x),
$$

then

$$
f(x) \Psi^{p-\gamma}(x) \leq f^{p-\gamma+1}(x) \Lambda^{p-\gamma}(x) .
$$

Using the chain rule (1.9) and the fact that $\nabla_{a}^{\gamma}(\Psi(x))=\lambda(x) f(x) \geq 0$, we get

$$
\begin{aligned}
\nabla_{a}^{\gamma}\left(\Psi^{p-\gamma+1}(x)\right) & =(p-\gamma+1) \nabla_{a}^{\gamma}(\Psi(x)) \int_{0}^{1}[h \Psi(\rho(x))+(1-h) \Psi(x)]^{p-\gamma} d h \\
& \leq(p-\gamma+1) \lambda(x) f(x) \int_{0}^{1}[h \Psi(x)+(1-h) \Psi(x)]^{p-\gamma} d h \\
& =(p-\gamma+1) \lambda(x) f(x) \Psi^{p-\gamma}(x) .
\end{aligned}
$$

Combining (2.14) with (2.15) gives

$$
\nabla_{a}^{\gamma}\left(\Psi^{p-\gamma+1}(x)\right) \leq(p-\gamma+1) \lambda(x) \Lambda^{p-\gamma}(x) f^{p-\gamma+1}(x)
$$

and thus

$$
\frac{\lambda(t) \nabla_{a}^{\gamma}\left(\Psi^{p-\gamma+1}(x)\right)}{\Lambda^{\beta}(t)} \leq \frac{(p-\gamma+1) \lambda(t) \lambda(x) \Lambda^{p-\gamma}(x) f^{p-\gamma+1}(x)}{\Lambda^{\beta}(t)} .
$$

Therefore,

$$
\begin{aligned}
\frac{\lambda(t) \nabla_{a}^{\gamma} \Psi^{p-\gamma+1}(t)}{\Lambda^{\beta}(t)} & =\int_{r}^{t} \frac{\lambda(t) \nabla_{a}^{\gamma} \Psi^{p-\alpha+1}(x)}{\Lambda(t)^{\beta}} \nabla_{a}^{\gamma} x \\
& \leq(p-\gamma+1) \int_{r}^{t} \frac{\lambda(t) \lambda(x) \Lambda^{p-\gamma}(x) f^{p-\gamma+1}(x)}{\Lambda^{\beta}(t)} \nabla_{a}^{\gamma} x,
\end{aligned}
$$

and hence

$$
\begin{aligned}
& \int_{r}^{\infty} \frac{\lambda(t) \Psi^{p-\gamma+1}(t)}{\Lambda^{\beta}(t)} \nabla_{a}^{\gamma} t \\
& \quad \leq(p-\gamma+1) \int_{r}^{\infty}\left[\int_{r}^{t} \frac{\lambda(t) \lambda(x) \Lambda^{p-\gamma}(x) f^{p-\gamma+1}(x)}{\Lambda^{\beta}(t)} \nabla_{a}^{\gamma} x\right] \nabla_{a}^{\gamma} t
\end{aligned}
$$

By making use of Fubini's theorem on time scales, inequality (2.16) can be rewritten as

$$
\begin{aligned}
& \int_{r}^{\infty} \frac{\lambda(t) \Psi^{p-\gamma+1}(t)}{\Lambda^{\beta}(t)} \nabla_{a}^{\gamma} t \\
& \quad \leq(p-\gamma+1) \int_{r}^{\infty} \lambda(x) \Lambda^{p-\gamma}(x) f^{p-\gamma+1}(x)\left[\int_{x}^{\infty} \lambda(t) \Lambda^{-\beta}(t) \nabla_{a}^{\gamma} t\right] \nabla_{a}^{\gamma} x .
\end{aligned}
$$

Again using the chain rule (1.10), there is $c \in[\rho(t), t]$ such that

$$
\nabla_{a}^{\gamma} \Lambda^{-\beta+1}(t)=(1-\beta) \Lambda^{-\beta}(c) \nabla_{a}^{\gamma} \Lambda(t)
$$




$$
\geq(1-\beta) \lambda(t) \Lambda^{-\beta}(t) .
$$

Substituting (2.18) into (2.17) gives

$$
\begin{aligned}
& \int_{r}^{\infty} \frac{\lambda(t) \Psi^{p-\gamma+1}(t)}{\Lambda^{\beta}(t)} \nabla_{a}^{\gamma} t \\
& \quad \leq \frac{p-\gamma+1}{1-\beta} \int_{r}^{\infty} \lambda(x) \Lambda^{p-\gamma}(x) f^{p-\gamma+1}(x)\left[\int_{x}^{\infty} \nabla_{a}^{\gamma} \Lambda^{-\beta+1}(t) \nabla_{a}^{\gamma} t\right] \nabla_{a}^{\gamma} x \\
& \quad=\frac{p-\gamma+1}{\beta-1} \int_{r}^{\infty} \lambda(x) \Lambda^{p-\gamma-\beta+1}(x) f^{p-\gamma+1}(x) \nabla_{a}^{\gamma} x .
\end{aligned}
$$

This completes the proof.

Again, we present some special cases of our results to the continuous, discrete, and quantum $\alpha$-conformable inequalities. Namely, in cases of time scales $\mathbb{T}=\mathbb{R}, \mathbb{T}=h \mathbb{Z}, \mathbb{T}=\mathbb{Z}$, and $\mathbb{T}=q^{\mathbb{N}_{0}}$.

Corollary 2.12 If $\mathbb{T}=\mathbb{R}$ in Theorem 2.11 , inequality (2.13) reduces to

$$
\int_{r}^{\infty} \frac{\lambda(t) \Psi^{p-\gamma+1}(t)}{\Lambda^{\beta}(t)}(t-a)^{\gamma-1} d t \leq \frac{p-\gamma+1}{\beta-1} \int_{r}^{\infty} \lambda(t) \Lambda^{p-\beta}(t) f^{p-\gamma+1}(t)(t-a)^{\alpha-1} d t
$$

where

$$
\Psi(t)=\int_{r}^{t} \lambda(s) f(s)(s-a)^{\gamma-1} d s \quad \text { and } \quad \Lambda(t)=\int_{r}^{t} \lambda(s)(s-a)^{\gamma-1} d s .
$$

Corollary 2.13 If $\mathbb{T}=h \mathbb{Z}$ in Theorem 2.11 , inequality (2.13) reduces to

$$
\begin{aligned}
& \sum_{t=\frac{r}{h}}^{\infty} \frac{\lambda(h t) \Psi^{p-\gamma+1}(h t)}{\Lambda^{\beta}(h t)}\left(\rho^{\gamma-1}(h t)-a\right)_{h}^{(\gamma-1)} \\
& \quad \leq \frac{p-\gamma+1}{\beta-1} \sum_{t=\frac{r}{h}}^{\infty} \lambda(h t) \Lambda^{p-\beta}(h t) f^{p-\gamma+1}(h t)\left(\rho^{\gamma-1}(h t)-a\right)_{h}^{(\gamma-1)},
\end{aligned}
$$

where

$$
\begin{aligned}
& \Psi(t)=h \sum_{s=\frac{r}{h}}^{\frac{t}{h}-1} \lambda(h s) f(h s)\left(\rho^{\gamma-1}(h t)-a\right)_{h}^{(\gamma-1)} \text { and } \\
& \Lambda(t)=h \sum_{s=\frac{r}{h}}^{\frac{t}{h}-1} \lambda(h s)\left(\rho^{\gamma-1}(h t)-a\right)_{h}^{(\gamma-1)} .
\end{aligned}
$$

Corollary 2.14 For $\mathbb{T}=\mathbb{Z}$, we simply take $h=1$ in Corollary 2.13. In this case, inequality (2.13) reduces to

$$
\sum_{t=r}^{\infty} \frac{\lambda(t) \Psi^{p-\gamma+1}(t)}{\Lambda^{\beta}(t)}\left(\rho^{\gamma-1}(t)-a\right)^{(\gamma-1)}
$$




$$
\leq \frac{p-\gamma+1}{\beta-1} \sum_{t=r}^{\infty} \lambda(t) \Lambda^{p-\beta}(t) f^{p-\gamma+1}(t)\left(\rho^{\gamma-1}(t)-a\right)^{(\gamma-1)}
$$

where

$$
\Psi(t)=\sum_{s=r}^{t-1} \lambda(s) f(s)\left(\rho^{\gamma-1}(s)-a\right)^{(\gamma-1)} \quad \text { and } \quad \Lambda(t)=\sum_{s=r}^{t-1} \lambda(s)\left(\rho^{\gamma-1}(s)-a\right)^{(\gamma-1)} .
$$

Corollary 2.15 If $\mathbb{T}=q^{\mathbb{N}_{0}}$ in Theorem 2.11, then inequality (2.13) reduces to

$$
\begin{aligned}
& \sum_{t \in(r, \infty)} \frac{t \lambda(t) \Psi^{p-\gamma+1}(t)}{\Lambda^{\beta}(t)}\left(\rho^{\gamma-1}(t)-a\right)_{\tilde{q}}^{(\gamma-1)} \\
& \quad \leq \frac{p-\gamma+1}{\beta-1} \sum_{t \in(r, t)} t \lambda(t) \Lambda^{p-\beta}(t) f^{p-\gamma+1}(t)\left(\rho^{\gamma-1}(t)-a\right)_{\tilde{q}}^{(\gamma-1)},
\end{aligned}
$$

where

$$
\begin{aligned}
& \Psi(t)=(\tilde{q}-1) \sum_{s \in(r, t)} s \lambda(s) f(s)\left(\rho^{\gamma-1}(s)-a\right)_{\tilde{q}}^{(\gamma-1)} \text { and } \\
& \Lambda(t)=(\tilde{q}-1) \sum_{s \in(r, t)} s \lambda(s)\left(\rho^{\gamma-1}(s)-a\right)_{\tilde{q}}^{(\gamma-1)} .
\end{aligned}
$$

We next discuss inequality (2.13) for the case when the limit of integral $\int_{r}^{t} \lambda(s) \nabla_{a}^{\gamma} s$ is changed from $t$ to $\infty$.

Theorem 2.16 Under the same hypotheses of Theorem 2.1 with $\beta \geq 1$, then we have that

$$
\int_{r}^{\infty} \frac{\lambda(t) \Psi^{p-\gamma+1}(t)}{\left(\Omega^{\rho}(t)\right)^{\beta}} \nabla_{a}^{\gamma} t \leq \frac{p-\gamma+1}{1-\beta} \int_{r}^{\infty} \lambda(x) \Lambda^{p-\gamma}(t) \Omega^{1-\beta}(t) f^{p-\gamma+1}(t) \nabla_{a}^{\gamma} t,
$$

where

$$
\Psi(t)=\int_{r}^{t} \lambda(s) f(s) \nabla_{a}^{\gamma} s, \quad \Lambda(t)=\int_{r}^{t} \lambda(s) \nabla_{a}^{\gamma} s \quad \text { and } \quad \Omega(t)=\int_{t}^{\infty} \lambda(s) \nabla_{a}^{\gamma} s .
$$

Proof Since $f$ is nondecreasing, we have for $x \geq r$

$$
\begin{aligned}
\Psi(x) & =\int_{r}^{x} \lambda(s) f(s) \nabla_{a}^{\gamma} s \\
& \leq f(x) \int_{r}^{x} \lambda(s) \nabla_{a}^{\gamma} s=f(x) \Lambda(x),
\end{aligned}
$$

then

$$
f(x) \Psi^{p-\gamma}(x) \leq f^{p-\gamma+1}(x) \Lambda^{p-\gamma}(x) .
$$

Employing the chain rule (1.9) and using $\nabla_{a}^{\gamma} \Psi(x)=\lambda(x) f(x) \geq 0$, we get

$$
\nabla_{a}^{\gamma}\left(\Psi^{p-\gamma+1}(x)\right)=(p-\gamma+1) \nabla_{a}^{\gamma} \Psi(x) \int_{0}^{1}[h \Psi(\rho(x))+(1-h) \Psi(x)]^{p-\alpha} d h
$$




$$
\begin{aligned}
& \leq(p-\gamma+1) \lambda(x) f(x) \int_{0}^{1}[h \Psi(x)+(1-h) \Psi(x)]^{p-\gamma} d h \\
& =(p-\gamma+1) \lambda(x) f(x) \Psi^{p-\gamma}(x) .
\end{aligned}
$$

Combining (2.20) with (2.21) leads to

$$
\nabla_{a}^{\gamma}\left(\Psi^{p-\gamma+1}(x)\right) \leq(p-\gamma+1) \lambda(x) \Lambda^{p-\gamma}(x) f^{p-\gamma+1}(x),
$$

and so

$$
\frac{\lambda(t) \nabla_{a}^{\gamma}\left(\Psi^{p-\gamma+1}(x)\right)}{\left(\Omega^{\rho}(t)\right)^{\beta}} \leq \frac{(p-\gamma+1) \lambda(t) \lambda(x) \Lambda^{p-\gamma}(x) f^{p-\gamma+1}(x)}{\left(\Omega^{\rho}(t)\right)^{\beta}} .
$$

Thus,

$$
\begin{aligned}
\frac{\lambda(t) \Psi^{p-\gamma+1}(t)}{\left(\Omega^{\rho}(t)\right)^{\beta}} & =\int_{r}^{t} \frac{\lambda(t) \nabla_{a}^{\gamma}\left(\Psi^{p-\gamma+1}(x)\right)}{\left(\Omega^{\rho}(t)\right)^{\beta}} \nabla_{a}^{\gamma} x \\
& \leq(p-\gamma+1) \int_{r}^{t} \frac{\lambda(t) \lambda(x) \Lambda^{p-\gamma}(x) f^{p-\gamma+1}(x)}{\left(\Omega^{\rho}(t)\right)^{\beta}} \nabla_{a}^{\gamma} x,
\end{aligned}
$$

and hence

$$
\begin{aligned}
& \int_{r}^{\infty} \frac{\lambda(t) \Psi^{p-\gamma+1}(t)}{\left(\Omega^{\rho}(t)\right)^{\beta}} \nabla_{a}^{\gamma} t \\
& \quad \geq(p-\gamma+1) \int_{r}^{\infty}\left[\int_{r}^{t} \frac{\lambda(t) \lambda(x) \Lambda^{p-\gamma}(x) f^{p-\gamma+1}(x)}{\left(\Omega^{\rho}(t)\right)^{\beta}} \nabla_{a}^{\gamma} x\right] \nabla_{a}^{\gamma} t .
\end{aligned}
$$

Employing Fubini's theorem on time scales, inequality (2.22) can be rewritten as

$$
\begin{aligned}
& \int_{r}^{\infty} \frac{\lambda(t) \Psi^{p-\gamma+1}(t)}{\left(\Omega^{\rho}(t)\right)^{\beta-\gamma+1}} \nabla_{a}^{\gamma} t \\
& \quad \leq(p-\gamma+1) \int_{r}^{\infty} \lambda(x) \Lambda^{p-\gamma}(x) f^{p-\gamma+1}(x)\left[\int_{x}^{\infty} \lambda(t)\left(\Omega^{\rho}(t)\right)^{-\beta} \nabla_{a}^{\gamma} t\right] \nabla_{a}^{\gamma} x .
\end{aligned}
$$

We employ the chain rule (1.10) again to have $d \in[\rho(t), t]$ such that

$$
\nabla_{a}^{\gamma} \Omega^{-\beta+1}(t)=(1-\beta) \Omega^{-\beta}(d) \nabla_{a}^{\gamma} \Omega(t) \geq(\beta-1) \lambda(t)\left(\Omega^{\rho}(t)\right)^{-\beta} .
$$

Substituting (2.24) into (2.23) yields

$$
\begin{aligned}
\int_{r}^{\infty} & \frac{\lambda(t) \Psi^{p-\gamma+1}(t)}{\left(\Omega^{\rho}(t)\right)^{\beta}} \nabla_{a}^{\gamma} t \\
& \leq \frac{p-\gamma+1}{\beta-1} \int_{r}^{\infty} \lambda(x) \Lambda^{p-\gamma}(x) f^{p-\gamma+1}(x)\left[\int_{x}^{\infty} \nabla_{a}^{\gamma}\left(\Omega^{1-\beta}(t)\right) \nabla_{a}^{\gamma} t\right] \nabla_{a}^{\gamma} x \\
\quad & \frac{p-\gamma+1}{1-\beta} \int_{r}^{\infty} \lambda(x) \Lambda^{p-\gamma}(x) \Omega^{1-\beta}(x) f^{p-\gamma+1}(x) \nabla_{a}^{\gamma} x,
\end{aligned}
$$

which is our desired inequality (2.19). 
Now, as special cases of our results, we will give the continuous, discrete, and quantum $\alpha$-conformable inequalities. Namely, in cases of time scales $\mathbb{T}=\mathbb{R}, \mathbb{T}=h \mathbb{Z}, \mathbb{T}=\mathbb{Z}$, and $\mathbb{T}=q^{\mathbb{N}_{0}}$.

Corollary 2.17 If $\mathbb{T}=\mathbb{R}$ in Theorem 2.16, then inequality (2.19) boils down to

$$
\begin{aligned}
& \int_{r}^{\infty} \frac{\lambda(t) \Psi^{p-\gamma+1}(t)}{\Omega^{\beta}(t)}(t-a)^{\gamma-1} d t \\
& \quad \leq \frac{p-\gamma+1}{1-\beta} \int_{r}^{\infty} \lambda(t) \Lambda^{p-\gamma}(t) \Omega^{\gamma-\beta}(t) f^{p-\gamma+1}(t)(t-a)^{\gamma-1} d t,
\end{aligned}
$$

where

$$
\begin{aligned}
& \Psi(t)=\int_{r}^{t} \lambda(s) f(s)(s-a)^{\gamma-1} d s, \quad \Lambda(t)=\int_{r}^{t} \lambda(s)(s-a)^{\gamma-1} d s \text { and } \\
& \Omega(t)=\int_{t}^{\infty} \lambda(s)(s-a)^{\gamma-1} d s .
\end{aligned}
$$

Corollary 2.18 If $\mathbb{T}=h \mathbb{Z}$ in Theorem 2.16, then inequality (2.19) boils down to

$$
\begin{aligned}
& \sum_{t=\frac{r}{h}}^{\infty} \frac{\lambda(h t) \Psi^{p-\gamma+1}(h t)}{\Omega^{\beta}(h t-h)}\left(\rho(h t)^{\gamma-1}-a\right)_{h}^{(1-\gamma)} \\
& \quad \leq \frac{p-\gamma+1}{1-\beta} \sum_{t=\frac{r}{h}}^{\infty} \lambda(h t) \Lambda^{p-\gamma}(h t) \Omega^{\gamma-\beta}(h t) f^{p-\gamma+1}(h t)\left(\rho(h t)^{\gamma-1}-a\right)_{h}^{(1-\gamma)},
\end{aligned}
$$

where

$$
\begin{aligned}
& \Psi(t)=h \sum_{s=\frac{r}{h}}^{\frac{t}{h}-1} \lambda(h s) f(h s)\left(\rho(h s)^{\gamma-1}-a\right)_{h}^{(1-\gamma)}, \\
& \Lambda(t)=h \sum_{s=\frac{r}{h}}^{\frac{t}{h}-1} \lambda(h s)\left(\rho(h s)^{\gamma-1}-a\right)_{h}^{(1-\gamma)} \text { and } \\
& \Omega(t)=h \sum_{s=\frac{t}{h}}^{\infty} \lambda(h s)\left(\rho(h s)^{\gamma-1}-a\right)_{h}^{(1-\gamma)} .
\end{aligned}
$$

Corollary 2.19 For $\mathbb{T}=\mathbb{Z}$, we simply take $h=1$ in Corollary 2.18. In this case, inequality (2.19) boils down to

$$
\begin{aligned}
& \sum_{t=r}^{\infty} \frac{\lambda(t) \Psi^{p-\gamma+1}(t)}{\Omega^{\beta}(t-1)}\left(\rho(t)^{\gamma-1}-a\right)^{(1-\gamma)} \\
& \quad \leq \frac{p-\gamma+1}{1-\beta} \sum_{t=r}^{\infty} \lambda(t) \Lambda^{p-\gamma}(t) \Omega^{\gamma-\beta}(t) f^{p-\gamma+1}(t)\left(\rho(t)^{\gamma-1}-a\right)^{(1-\gamma)},
\end{aligned}
$$


where

$$
\begin{aligned}
& \Psi(t)=\sum_{s=r}^{t-1} \lambda(s) f(s)\left(\rho(s)^{\gamma-1}-a\right)^{(1-\gamma)}, \quad \Lambda(t)=\sum_{s=r}^{t-1} \lambda(s)\left(\rho(s)^{\gamma-1}-a\right)^{(1-\gamma)} \text { and } \\
& \Omega(t)=\sum_{s=t}^{\infty} \lambda(s)\left(\rho(h s)^{\gamma-1}-a\right)^{(1-\gamma)} .
\end{aligned}
$$

Corollary 2.20 If $\mathbb{T}=q^{\mathbb{N}_{0}}$ in Theorem 2.16, inequality (2.19) boils down to

$$
\begin{aligned}
& \sum_{t \in(r, \infty)} \frac{t \lambda(t) \Psi^{p-\gamma+1}(t)}{\Omega^{\beta}(\rho(t))}\left(\rho(t)^{\gamma-1}-a\right)_{\tilde{q}}^{(1-\gamma)} \\
& \quad \leq \frac{p-\gamma+1}{1-\beta} \sum_{t \in(r, \infty)} t \lambda(t) \Lambda^{p-\gamma}(t) \Omega^{\gamma-\beta}(t) f^{p-\gamma+1}(t)\left(\rho(t)^{\gamma-1}-a\right)_{\tilde{q}}^{(1-\gamma)},
\end{aligned}
$$

where

$$
\begin{aligned}
& \Psi(t)=(\tilde{q}-1) \sum_{s \in(r, t)} s \lambda(s) f(s)\left(\rho(s)^{\gamma-1}-a\right)_{\tilde{q}}^{(1-\gamma)}, \\
& \Lambda(t)=(\tilde{q}-1) \sum_{s \in[r, t]} s \lambda(s)\left(\rho(s)^{\gamma-1}-a\right)_{\tilde{q}}^{(1-\gamma)} \text { and } \\
& \Omega(t)=(\tilde{q}-1) \sum_{s \in(r, \infty)} s \lambda(s)\left(\rho(s)^{\gamma-1}-a\right)_{\tilde{q}}^{(1-\gamma)} .
\end{aligned}
$$

\section{Conclusion}

In this important work, we discussed some new dynamic inequalities of Hardy type using nabla integral on time scales. By employing the conformable fractional $\nabla$-conformableintegral on time scales, several $\nabla$-conformable Hardy-type inequalities on time scales have been proved. Our proposed results show the potential for producing some original continuous, discrete, and quantum inequalities. We further presented some relevant inequalities as special cases: discrete inequalities and integral inequalities. These results may be used to obtain more generalized results of several obtained inequalities before.

Acknowledgements

Not applicable yet.

Funding

Not applicable.

Availability of data and materials

Not applicable.

\section{Declarations}

Competing interests

The authors announce that there are not any competing interests.

Authors' contributions

All authors have read and finalized the manuscript with equal contribution. 


\section{Author details}

${ }^{1}$ Department of Mathematics, Faculty of Science, Al-Azhar University, Nasr City (11884), Cairo, Egypt. ${ }^{2}$ Department of Mathematics and Computer Science, Alabama State University, Montgomery, AL, USA. ${ }^{3}$ Department of Mathematics, Clarkson University, Potsdam, NY, USA. ${ }^{4}$ Department of Mathematics, Cankaya University, Ankara, 06530, Turkey. ${ }^{5}$ Institute of Space Science, Magurele-Bucharest, Romania. ${ }^{6}$ Department of Medical Research, China Medical University Hospital, China Medical University, Taichung, Taiwan.

\section{Publisher's Note}

Springer Nature remains neutral with regard to jurisdictional claims in published maps and institutional affiliations.

Received: 20 September 2021 Accepted: 8 November 2021 Published online: 09 December 2021

\section{References}

1. Abd El-Hamid, H.A., Rezk, H.M., Ahmed, A.M., AlNemer, G., Zakarya, M., El Saify, H.A.: Some dynamic Hilbert-type inequalities for two variables on time scales. J. Inequal. Appl. 2021(1), 1 (2021)

2. Abdel-Moneim El-Deeb, A., Bazighifan, O., Awrejcewicz, J.: A variety of dynamic Steffensen-type inequalities on a general time scale. Symmetry 13(9), 1738 (2021)

3. Abdeldaim, A., El-Deeb, A.A.: On generalized of certain retarded nonlinear integral inequalities and its applications in retarded integro-differential equations (2015)

4. Abdeljawad, T:: On conformable fractional calculus. J. Comput. Appl. Math. 279, 57-66 (2015)

5. Adil Khan, M., Ali, T., Dragomir, S.S., Sarikaya, M.Z:: Hermite-Hadamard type inequalities for conformable fractional integrals. Rev. R. Acad. Cienc. Exactas Fís. Nat., Ser. A Mat. 112(4), 1033-1048 (2018)

6. Agarwal, R.P., Bohner, M., Peterson, A.: Inequalities on time scales: a survey. Math. Inequal. Appl. 4(4), 535-557 (2001)

7. Agarwal, R.P., Mahmoud, R.R., O'Regan, D., Aker, S.H.: Some reverse dynamic inequalities on time scales. Bull. Aust. Math. Soc. 96(3), 445-454 (2017)

8. Agarwal, R.P., O'Regan, D., Saker, S.H.: Hardy Type Inequalities on Time Scales. Springer, Cham (2016)

9. AlNemer, G., Kenawy, M., Zakarya, M., Cesarano, C., Rezk, H.M.: Generalizations of Hardy's type inequalities via conformable calculus. Symmetry 13(2), 242 (2021)

10. Asarikaya, M.Z., Billisik, C.C.: Opial type inequalities for conformable fractional integrals via convexity

11. Bendouma, B., Hammoudi, A.: A nabla conformable fractional calculus on time scales. Electron. J. Math. Anal. Appl. 7(1), 202-216 (2019)

12. Bohner, M., Peterson, A.: Dynamic Equations on Time Scales: An Introduction with Applications. Birkhäuser, Boston (2001)

13. Bohner, M., Peterson, A. (eds.): Advances in Dynamic Equations on Time Scales Birkhäuser, Boston (2003)

14. Čermák, J., Nechvátal, L.: On (q, h)-analogue of fractional calculus. J. Nonlinear Math. Phys. 17(01), 51-68 (2010)

15. Chu, Y., Khan, M.A., Ali, T., Dragomir, S.S.: Inequalities for $\alpha$-fractional differentiable functions. J. Inequal. Appl. 2017(1), 1 (2017)

16. Copson, E.T.: Note on series of positive terms. J. Lond. Math. Soc. 3(1), 49-51 (1928)

17. Daftardar-Gejji, V., Jafari, H.: Analysis of a system of nonautonomous fractional differential equations involving Caputo derivatives. J. Math. Anal. Appl. 328(2), 1026-1033 (2007)

18. Donchev, T., Nosheen, A., Pečarić, J.: Hardy-type inequalities on time scale via convexity in several variables. ISRN Math. Anal. (2013)

19. El-Deeb, A.A.: Some Gronwall-Bellman type inequalities on time scales for Volterra-Fredholm dynamic integral equations. J. Egypt. Math. Soc. 26(1), 1-17 (2018)

20. El-Deeb, A.A.: A variety of nonlinear retarded integral inequalities of Gronwall type and their applications. In: Advances in Mathematical Inequalities and Applications, pp. 143-164. Springer, Berlin (2018)

21. El-Deeb, A.A., Baleanu, D.: New weighted Opial-type inequalities on time scales for convex functions. Symmetry 12(5), $842(2020)$

22. El-Deeb, A.A., Bazighifan, O., Awrejcewicz, J.: On some new weighted Steffensen-type inequalities on time scales. Mathematics 9(21), 2670 (2021)

23. El-Deeb, A.A., El-Sennary, H.A., Khan, Z.A.: Some reverse inequalities of Hardy type on time scales. Adv. Differ. Equ. 2020(1), 1 (2020)

24. El-Deeb, A.A., Makharesh, S.D., Baleanu, D.: Dynamic Hilbert-type inequalities with Fenchel-Legendre transform. Symmetry 12(4), $582(2020)$

25. Hardy, G.H.: Note on a theorem of Hilbert. Math. Z. 6(3-4), 314-317 (1920)

26. Hardy, G.H.: Notes on some points in the integral calculus (LX). Messenger Math. 54, 150-156 (1925)

27. Hilger, S.: Analysis on measure chains - a unified approach to continuous and discrete calculus. Results Math. $18(1-2), 18-56(1990)$

28. Hilscher, R.: A time scales version of a Wirtinger-type inequality and applications. J. Comput. Appl. Math. 141(1-2), 219-226 (2002)

29. Iyiola, O.S., Nwaeze, E.R.: Some new results on the new conformable fractional calculus with application using d'Alambert approach. Prog. Fract. Differ. Appl. 2(2), 115-122 (2016)

30. Iyiola, O.S., Ojo, G.O.: On the analytical solution of Fornberg-Whitham equation with the new fractional derivative Pramana 85(4), 567-575 (2015)

31. Khalil, R., Al Horani, M., Yousef, A., Sababheh, M.: A new definition of fractional derivative. J. Comput. Appl. Math. 264, 65-70 (2014)

32. Kilbas, A., Srivastava, H., Trujillo, J.: Theory and Applications of Fractional Differential Equations, vol. 204. Elsevier, Amsterdam (2006)

33. Miller, K.S., Ross, B.: An Introduction to the Fractional Calculus and Fractional Differential Equations. Wiley, New York (1993) 
34. Nwaeze, E.R., Torres, D.F.M.: Chain rules and inequalities for the BHT fractional calculus on arbitrary timescales. Arab. J. Math. 6(1), 13-20 (2017)

35. Oguntuase, J.A., Persson, L.-E.: Time scales Hardy-type inequalities via superquadracity. Ann. Funct. Anal. 5(2), 61-73 (2014)

36. Ozkan, U.M., Yildirim, H.: Hardy-Knopp-type inequalities on time scales. Dyn. Syst. Appl. 17(3-4), 477-486 (2008)

37. Podlubny, I.: Fractional Differential Equations: An Introduction to Fractional Derivatives, Fractional Differential Equations, to Methods of Their Solution and Some of Their Applications. Elsevier, Amsterdam (1998)

38. Rahmat, M.R.S., Salmi, M., Noorani, M.: A new conformable nabla derivative and its application on arbitrary time scales. Adv. Differ. Equ. 2021(1), 1 (2021)

39. Řehák, P: Hardy inequality on time scales and its application to half-linear dynamic equations. J. Inequal. Appl. 5, 495-507 (2005)

40. Renaud, P.F.: A reversed Hardy inequality. Bull. Aust. Math. Soc. 34(2), 225-232 (1986)

41. Rezk, H.M., AlNemer, G., El-Hamid, H.A.A., Abdel-Aty, A.-H., Nisar, K.S., Zakarya, M.: Hillbert-type inequalities for time scale nabla calculus. Adv. Differ. Equ. 2020(1), 1 (2020)

42. Saker, S.H., Kenawy, M., AlNemer, G.H., Zakarya, M.: Some fractional dynamic inequalities of Hardy's type via conformable calculus. Mathematics 8(3), 434 (2020)

43. Saker, S.H., O'Regan, D., Agarwal, R.: Generalized Hardy, Copson, Leindler and Bennett inequalities on time scales. Math. Nachr. 287(5-6), 686-698 (2014)

44. Saker, S.H., O'Regan, D., Agarwal, R.P.: Dynamic inequalities of Hardy and Copson type on time scales. Analysis 34(4), 391-402 (2014)

45. Sarikaya, M., Budak, H.: New inequalities of Opial type for conformable fractional integrals. Turk. J. Math. 41(5), 1164-1173 (2017)

46. Sarikaya, M., Yaldiz, H., Budak, H.: Steffensen's integral inequality for conformable fractional integrals. Int. J. Anal. Appl. 15(1), 23-30 (2017)

47. Set, E., Gözpınar, A., Ekinci, A.: Hermite-Hadamard type inequalities via comfortable fractional integrals. Acta Math. Univ. Comen. 86(2), 309-320 (2017)

48. Tasbozan, O., Kurt, A., Iyiola, O.S., Çenesiz, Y.: On the analytical solutions of the system of conformable time-fractional Robertson equations with 1-d diffusion. Chaos Solitons Fractals 94, 1-7 (2017)

49. Zakarya, M., Altanji, M., AlNemer, G.H., El-Hamid, A., Hoda, A., Cesarano, C., Rezk, H.M.: Fractional reverse Copson's inequalities via conformable calculus on time scales. Symmetry 13(4), 542 (2021)

\section{Submit your manuscript to a SpringerOpen ${ }^{\circ}$ journal and benefit from:}

- Convenient online submission

- Rigorous peer review

- Open access: articles freely available online

- High visibility within the field

- Retaining the copyright to your article

Submit your next manuscript at $\gg$ springeropen.com 